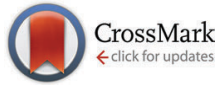

Cite this: J. Mater. Chem. B, 2015, 3, 6224

Received 13th May 2015, Accepted 15th June 2015

DOI: $10.1039 / c 5 t b 00906 e$

www.rsc.org/MaterialsB

\title{
Antimicrobial and cell-compatible surface- attached polymer networks - how the correlation of chemical structure to physical and biological data leads to a modified mechanism of action $\dagger$
}

\author{
Peng Zou, ${ }^{\mathrm{ab}}$ Dougal Laird, ${ }^{\mathrm{abc}}$ Esther K. Riga, ${ }^{a}$ Zhuoling Deng, ${ }^{\mathrm{ab}}$ Franziska Dorner, ${ }^{\mathrm{ab}}$ \\ Heidi-Rosalia Perez-Hernandez, ${ }^{\text {ab }}$ D. Lorena Guevara-Solarte, ${ }^{\text {ad }}$ \\ Thorsten Steinberg, ${ }^{\mathrm{C}}$ Ali Al-Ahmad ${ }^{\mathrm{d}}$ and Karen Lienkamp*ab
}

\begin{abstract}
We present a synthetic platform based on photo-induced thiol-ene chemistry, by which surfaceattached networks from antimicrobial poly(oxonorbornene) (so-called polymeric synthetic mimics of antimicrobial peptides, SMAMPs) could be easily obtained. By systematically varying hydrophobicity and charge density, surface-attached polymer networks with high antimicrobial activity and excellent cell compatibility were obtained. For the homopolymer networks with constant charge density, antimicrobial activity increased systematically with increasing hydrophobicity (i.e. decreasing swellability and apparent surface energy). Irrespective of charge density, the antimicrobial activity of all networks correlated with the acid constant $\mathrm{pK}$ and the isoelectric point (IEP) - the lower $\mathrm{p} K$ and IEP, the higher the antimicrobial activity. The cell compatibility of the networks increased with increasing swellability and apparent surface energy, and decreased with increasing charge density. The data corroborates that the mechanism of action of antimicrobial polymer surfaces depends on at least two mechanistic steps, one of which is hydrophobicity-driven and the other charge related. Therefore, we suggest a modified mechanistic model with a charge-driven and a hydrophobicity-driven step. For antimicrobial networks that only varied in hydrophobicity, the antimicrobial activities on surfaces and in solution also correlated - the higher the activity in solution, the higher the activity on surfaces. Thus, the hydrophobicity-driven step for activity on surfaces may be similar to the one in solution. Cell compatibility of SMAMPs in solution and on surfaces also showed a systematic positive correlation for all polymers, therefore this property also depends on the net hydrophobic balance of the polymer.
\end{abstract}

\section{Introduction}

Multi-resistant bacteria are a life-threatening issue worldwide. According to the World Health Organization (WHO), 'antibiotic resistance is no longer a prediction for the future; it is happening right now, across the world, and is putting at risk the ability to treat common infections in the community and hospitals. Without urgent, coordinated action, the world is heading towards

\footnotetext{
${ }^{a}$ Department of Microsystems Engineering (IMTEK), Albert-Ludwigs-Universität, Georges-Köhler-Allee 103, 79110 Freiburg, Germany.

E-mail: lienkamp@imtek.uni-freiburg.de

${ }^{b}$ Freiburg Institute for Advanced Studies (FRIAS), Albert-Ludwigs-Universität, Albertstr. 19, 79104 Freiburg, Germany

${ }^{c}$ Oral Biotechnology, University Medical Center of the Albert-Ludwigs-Universität, Hugstetter Str. 55, 79106 Freiburg, Germany

${ }^{d}$ Department of Operative Dentistry and Periodontology, Center for Dental Medicine of the Albert-Ludwigs-Universität, Hugstetter Str. 55, 79106 Freiburg, Germany

$\dagger$ Electronic supplementary information (ESI) available. See DOI: 10.1039/c5tb00906e
}

a post-antibiotic era, in which common infections and minor injuries, which have been treatable for decades, can once again kill'. ${ }^{1}$ Leading global health organizations identified major problems with antimicrobial resistance in $S$. aureus (hospital-acquired bloodstream, skin and surgical site infections), E. coli (particularly urinary tract infections) and $K$. pneumoniae (hospital-acquired bloodstream infections, pneumonia). ${ }^{1,2}$

Due to the severe infections caused by these 'superbugs', there is an ever-increasing demand for materials that restrain and eradicate them. Multi-resistant bacteria originate from exposure to sub-lethal antibiotic doses; in hospitals, they were found in the ambient atmosphere, on surgical equipment, and on patients' and medical staff's skin or clothing. ${ }^{3}$ From there, they proliferate and provide a microbe reservoir that defies even the most rigorous hygiene regime. ${ }^{3 b, 4}$ As a result, infections with resistant bacteria in hospitals are ever increasing. Patients that have received implants and catheterized patients have a particularly high infection risk, ${ }^{5}$ as only a few bacteria that contaminate a medical device surface 
can develop dangerous, antibiotic-resistant biofilms in less than 24 hours. $^{6}$ Thus, effective medical surfaces that prevent biofilm formation comprise an immediate need and, in addition to strict hygiene regimes, could help contain resistant bacteria.

There are numerous medical applications where contactkilling antimicrobial polymer surface coatings could meet that need. To be truly useful, they must be broad-band active against Gram-negative and Gram-positive bacteria, and non-toxic to the cells of the human organism. Additionally, they should not increase bacterial resistance. Synthetic mimics of antimicrobial peptides (SMAMPs) are polymers that are unlikely to increase bacterial resistance. ${ }^{7}$ These polymers mimic the structure of natural antimicrobial peptides (AMPs, Fig. 1a). ${ }^{8}$ Like their natural peptide archetypes, they are facially amphiphilic and act on bacteria through unspecific membrane damage or destruction. ${ }^{7 a, c, 9}$ However, because they are cationic, they distinguish between negatively charged bacteria, which are killed, and the cells of the host organism, which are overall charge neutral and therefore much less affected. This feature makes SMAMPs highly cell compatible and therefore ideal candidates for medical applications. Despite the substantial amount of work on cationic antimicrobial polymer surfaces in the literature, the mechanism of action of antimicrobial polymer surfaces and the effect of physical properties on their biological performance is still not fully understood. ${ }^{10}$ Examples of pioneering work are Klibanov and Tiller's systems, ${ }^{11}$ and work on poly(diallyl dimethylammonium), ${ }^{12}$ poly(butyl methacrylate-co-aminoethyl methylacrylate), ${ }^{13}$ and poly(2(dimethylamino) ethyl methacrylate)-based surfaces. ${ }^{10 b, 14}$ Additionally, recent work by Chan-Park, ${ }^{15}$ Tiller, ${ }^{16}$ and Busscher ${ }^{17}$ made significant contributions towards a mechanistic understanding.

In this paper, we describe how we translated the SMAMP concept from solution to materials. We synthesized three series of poly(oxonorbornene) SMAMPs with systematically varying hydrophobicity and charge density, ${ }^{7 d}$ which are key parameters for antimicrobial activity. We surface-immobilized these polymers by a newly developed, straight-forward process (Fig. 1b) and thus obtained three series of surface-attached antimicrobial polymer networks. We demonstrate that these poly(oxonorbornene)-based SMAMP networks make excellent antimicrobial coatings which, unlike most of the state-of-the-art, are broad-band active against bacteria and highly compatible with primary human fibroblast cells and immortalized human keratinocytes. Additionally, the coating process we describe is so simple that it can be extended onto virtually any 2D or 3D surface that carries hydroxyl groups. We proved this with a model catheter made from medical grade poly(dimethylsiloxane), showing that SMAMPs also work on real-life medical surfaces.

To further advance the mechanistic understanding of antimicrobial surfaces, we fully characterized and quantified the physical properties of our SMAMP networks and correlated these results to their biological properties. This gave additional insight on the relation between molecular design (charge density
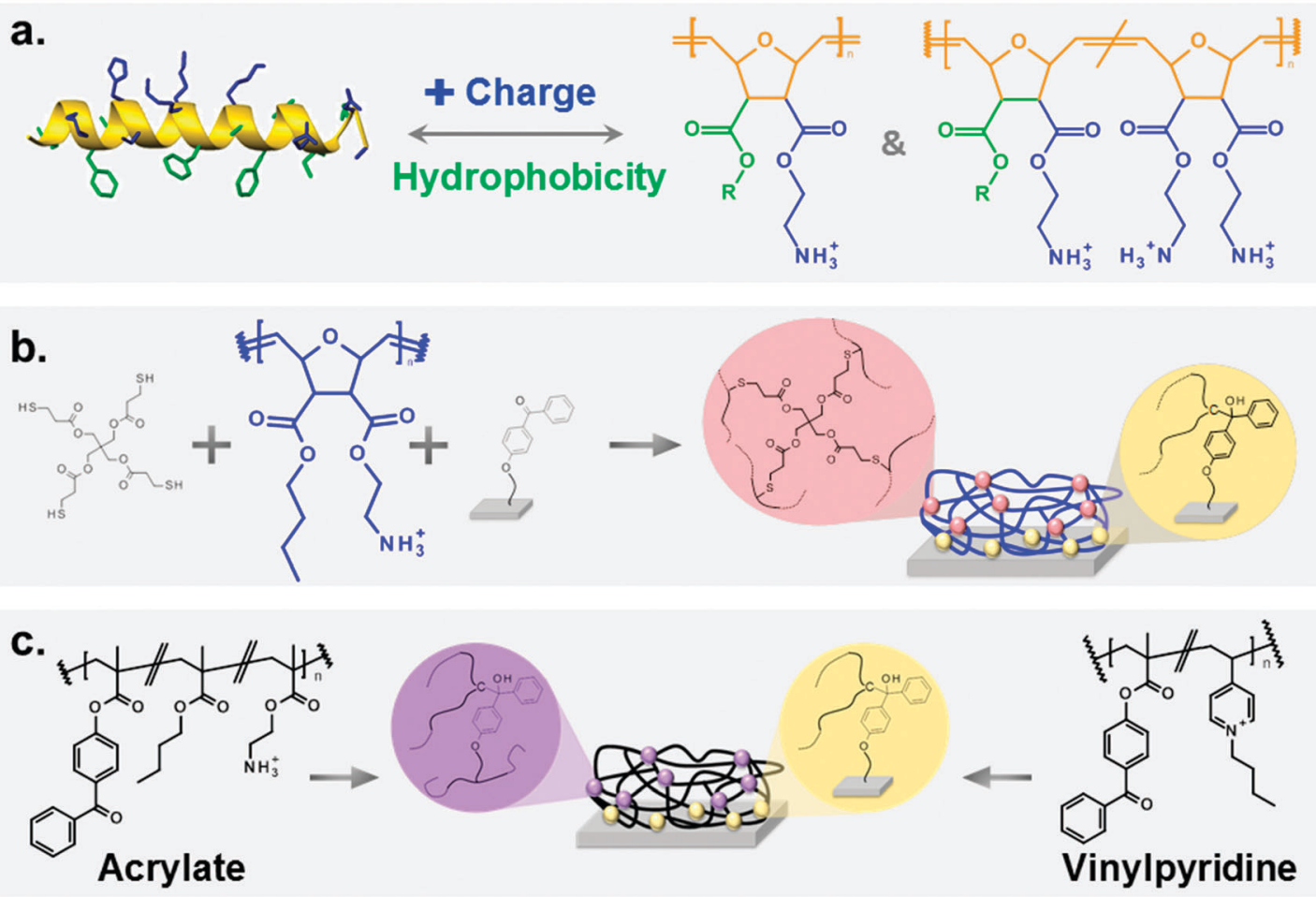

Fig. 1 (a) Antimicrobial peptides (AMPs, left) are the natural archetype for poly(oxanorbornene)-based synthetic mimics of antimicrobial peptides (SMAMPs, right). Both consist of hydrophobic (green) and cationic (blue) groups attached to a backbone (orange). Due to their facial amphiphilicity, these molecules are selectively active against bacteria. (b) SMAMPs (blue) were surface immobilized using a benzophenone anchor group, and crosslinked by pentaerithritol-tetrakis(3-mercaptopropionate) (= tetrathiol). As a result, antimicrobial surface-attached polymer networks were obtained. (c) Poly(methacrylate) and poly(vinylpyridine)-based surface-attached networks were synthesized as reference systems. 
and hydrophobicity), physical properties (zeta potential, swellability and surface energy) and biological performance (antimicrobial activity and cell compatibility). Moreover, we correlated the biological performance of our surface-attached SMAMP networks to the biological activities of structurally related SMAMPs in solution. In the light of the most recent discussion on the exact mechanism of cationic antimicrobial surfaces, this work may be an important contribution to the way we understand antimicrobial coatings.

\section{Results}

\section{Study design}

We chose poly(oxonorbornene) SMAMPs (Fig. 1a) as a synthetic platform that allowed systematic variation of two key parameters affecting antimicrobial activity: hydrophobicity and charge density. These molecules contain a variable substituent $\mathrm{R}$ and a positively charged group on each repeat unit. By changing $\mathrm{R}$ from methyl to butyl, homopolymers with variable hydrophobicity but constant nominal charge density were obtained. We also made copolymers containing variable amounts of a hydrophilic, doubly charged diamine repeat unit (Fig. 1a, right), and a hydrophobic repeat unit ( $\mathrm{R}=$ propyl or butyl, respectively). $\$$ Thus, one polymer series with a hydrophobicity gradient, and two series of copolymers with a simultaneous hydrophobicity and charge density gradient were obtained. This set of polymers enabled us to investigate the effects of gradual molecular changes on the macroscopic physical properties of the resulting polymer surfaces. At the same time, we could optimize the molecular parameters for optimal antimicrobial activity and cell compatibility.

The physical properties of these SMAMP networks were carefully characterized. We determined the apparent surface energy, the zeta potential, the swellability, and the surface morphology. The biological characterization included antimicrobial activity tests against 2 to 4 clinically relevant bacterial strains, and two toxicity tests. With this method pool, the present study probably comprises the most comprehensive characterization of antimicrobial polymer surfaces in literature.

Because it is notoriously difficult to compare antimicrobial data from different laboratories, we also included two antimicrobial systems from other groups as reference points. One is a poly(vinylpyrinine based) quaternary ammonium polymer, ${ }^{18}$ the other a poly(butyl methacrylate-co-ethanolamine methacrylate) system. ${ }^{19}$ In previous publications, these surfaces had brush-like architectures. For better comparability to our system, we also prepared them as surface-attached polymer networks (Fig. 1c).

\section{Synthesis}

The synthesis of the homopolymers and copolymers shown in Fig. 1a had been previously described for low molecular

\$ Our sample nomenclature indicates the repeat unit content of the copolymers. For example, P: D = 1: 9 designates a polymer with $10 \mathrm{~mol} \%$ propyl and $90 \mathrm{~mol} \%$ diamine repeat units. a.

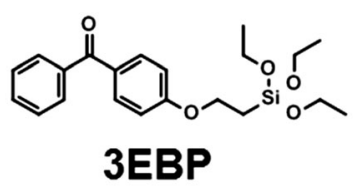

b.

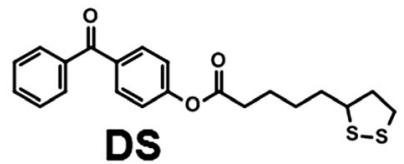

Fig. 2 Surface cross-linkers used in this study. (a) 3EBP forms selfassembled layers on any substrate that carries an $\mathrm{OH}$ group though $\mathrm{Si}-\mathrm{O}$ bonds; ${ }^{20}$ (b) DS attaches to gold via S-Au bonds to form a monolayer. ${ }^{21}$

weights, ${ }^{7 b-d}$ and was adjusted to obtain the higher molecular weights needed for the network formation. The surface coating process which we developed is shown in Fig. 1b. It makes use of the beautiful benzophenone chemistry that was introduced into the polymer field by Rühe and coworkers, ${ }^{20}$ which we combined with thiol-ene chemistry. To obtain surface-attached SMAMP networks, a solution of the SMAMP precursor polymer (with an N-Boc protective group) was mixed with the tetrathiol crosslinker shown in Fig. 1b, and spin-coated onto a benzophenonefunctionalized substrate. For most experiments, the substrate was a silicon wafer or glass slide functionalized with triethoxysilylbenzophenone (3EBP, Fig. 2a). For the surface plasmon resonance experiments, we used a gold surface functionalized with 4-hydroxybenzophenone-lipoic acid ester (DS, Fig. 2b). The benzophenone moiety in 3EBP and DS enabled the covalent link between the surface and adjacent polymer chains (yellow dots in Fig. 1b). The polymer-polymer cross-links were formed by the tetra-functional thiol cross-linker (pink dots in Fig. 1b). These two reactions were simultaneously activated by UV-irradiation, which initiated the $\mathrm{C}-\mathrm{H}$-insertion of the benzophenone, and the thiol-ene-reaction between the thiol cross-linker and the poly(oxonorbornene) double bond, respectively. The ratio of double bonds to $\mathrm{SH}$ groups was constant for all SMAMP networks to ensure an equal degree of cross-linking. The coatings also had approximately the same thickness (see Table 1 below). Keeping these two parameters constant allowed us to focus on the effect of structural changes in the repeat units on physical and biological properties. We activated the antimicrobial function of the SMAMP networks by converting the tert-butyl carbamate group of the precursor polymers into a primary ammonium group. The full process is described in the ESI† (pages 4-11, Schemes S1-S4).

A typical ${ }^{1} \mathrm{H}$-NMR spectrum and a representative GPC curve of the propyl SMAMP precursor are shown in Fig. 3a and b, respectively. The NMR spectrum of the propyl SMAMP precursor demonstrates the high purity of the polymer; the GPC data reveals a monomodal molecular weight distribution, with a molecular weight of $240000 \mathrm{~g} \mathrm{~mol}^{-1}$ and a polydispersity index of 1.1. The NMR and GPC data for all polymers are given in the ESI $\dagger$ (pages S8-S10). The chemical structure of the polymer networks was characterized by Attenuated Total Reflection 
Table 1 Physical characterization of surface-attached SMAMP networks. Dry layer thickness was measured by ellipsometry; the apparent surface energy was determined by the Zisman method; the swellability ratio was measured by surface plasmon resonance spectroscopy; the $\zeta$ potential parameters including $\mathrm{p} K$ were obtained by electrokinetic measurements

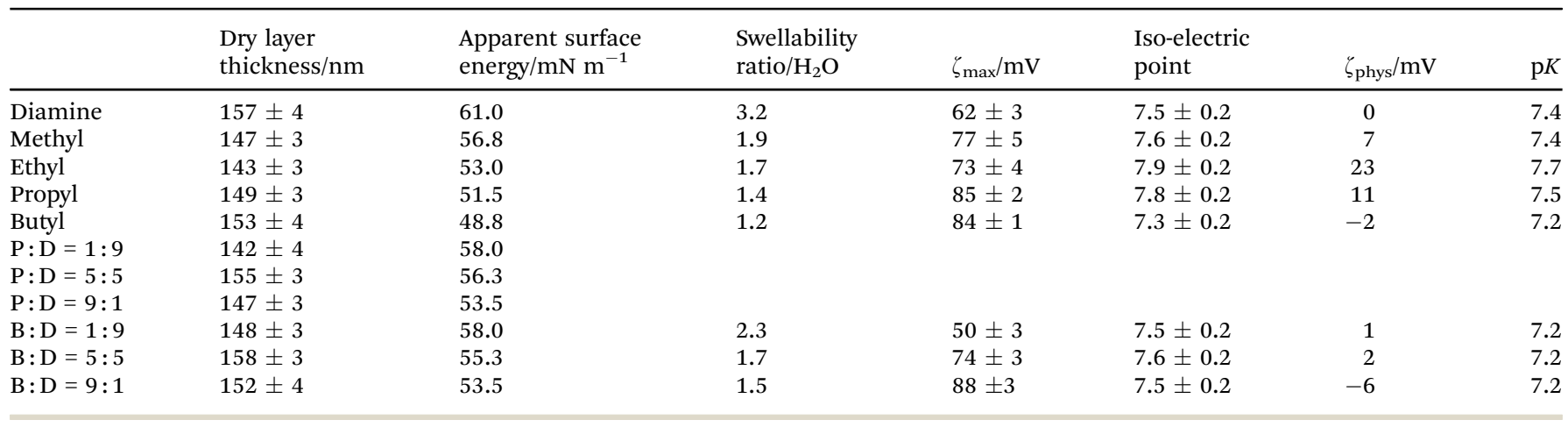
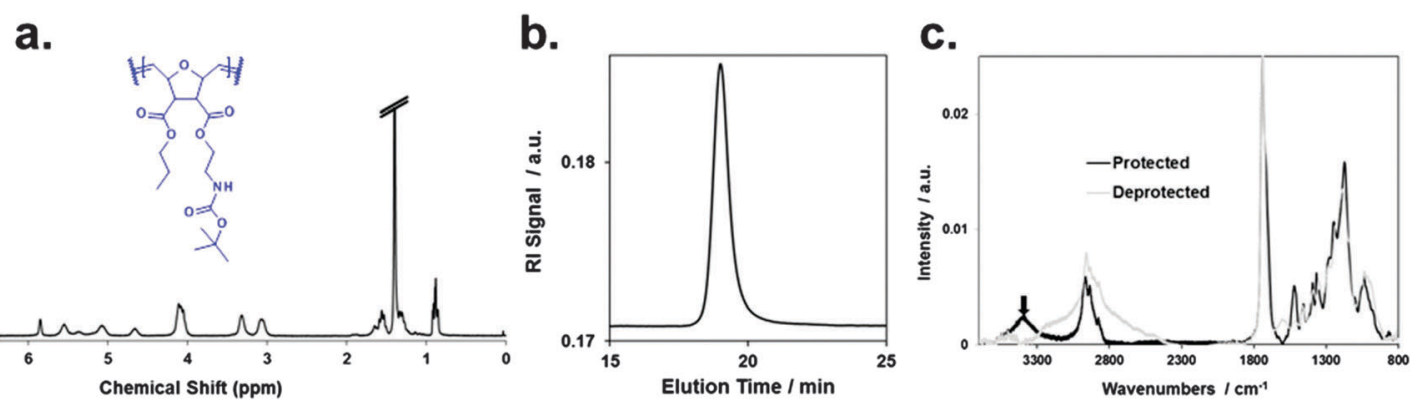

Fig. 3 Characterization of the propyl SMAMP precursor polymer and network: (a) ${ }^{1} \mathrm{H}-\mathrm{NMR}$ spectrum of the polymer, (b) gel permeation chromatography elugram of the polymer; (c) ATR-FTIR spectrum of the surface-attached precursor polymer network (protected) and activated polymer network (deprotected).

Fourier-transform Infrared Spectroscopy (ATR-FTIR, see ESI, $\dagger$ pages S12-S15). The ATR-FTIR spectrum of the propyl SMAMP precursor network and the deprotected, activated propyl SMAMP network are shown in Fig. 3c. The most prominent feature of the spectrum is the characteristic $\mathrm{C}=\mathrm{O}$ band at around $1750 \mathrm{~cm}^{-1}$, which is present in both compounds. After activation, the adsorption band at around $3400 \mathrm{~cm}^{-1}$ in the spectrum of the SMAMP precursor vanishes; this confirms successful removal of the N-Boc protective group. The formation of surface-attached polymer networks from the two antimicrobial reference polymers is illustrated in Fig. 1c. Covalent polymerpolymer cross-links in these reference samples were obtained through reactive methacryloxy benzophenone repeat units ${ }^{22}$ (purple dots in Fig. 1c), and polymer-surface cross-links through the 3EBP linker (yellow dots in Fig. 1c). The full synthetic procedure is given in the ESI $\dagger$ (page S29, Fig. S17).

\section{Physical characterization of the networks}

The physical characterization of the activated SMAMP networks is summarized in Table 1. All layers had a thickness in the range of 142 to $158 \mathrm{~nm}$. To quantify the relative hydrophobicity of each network, we measured the swelling ratioß in water using surface plasmon resonance/optical waveguide spectroscopy

$\S$ Swelling ratio = swollen layer thickness divided by dry layer thickness.
(SPR/OWG, Table 1). $\uparrow$ The SPR/OWG spectra of the networks are given in the ESI $\dagger$ (page S15, Table S6); the swelling ratios in water are given in Table 1. In the homopolymer series, the most hydrophilic network was the one with two diamine groups, with a swellability ratio of 3.2, followed by the methyl network with 1.9. The higher alkyl homologous had the expected lower swelling ratios. For the copolymer networks, the swellability also diminished systematically with increasing alkyl repeat unit content. The static and dynamic contact angles of all networks are given in the ESI $\dagger$ (page S18, Tables S7-S12). We quantified the surface energies using the Zisman method. $\|^{23}$ There are some limitations to this method - it is preferentially used for surfaces with low surface energies which do not swell in the

T In SPR/OWG, p-polarized light excites surface oscillations (plasmon and waveguide modes) in the gold layer, which result in light adsorption under certain angles of incidence. Thus, when measuring light intensity as a function of observation angle, several intensity minima (the plasmon and the waveguide modes) are obtained. These can be fitted to obtain the thickness and dielectric constants of the polymer layer on the gold surface. When the layer swells, the thickness and the dielectric constants change, which results in a shift of the plasmon and wave guide modes.

|| In this approach, the static contact angle (CA) of a test surface in contact with a series of solvent mixtures is measured. Plotting $\cos (\mathrm{CA})$ against the known surface energies of these solvent mixtures and extrapolation to $\cos (\mathrm{CA})=0$ gives the surface energy of a liquid that would fully wet the test surface, which is set equal to the surface energy of the test surface itself. 

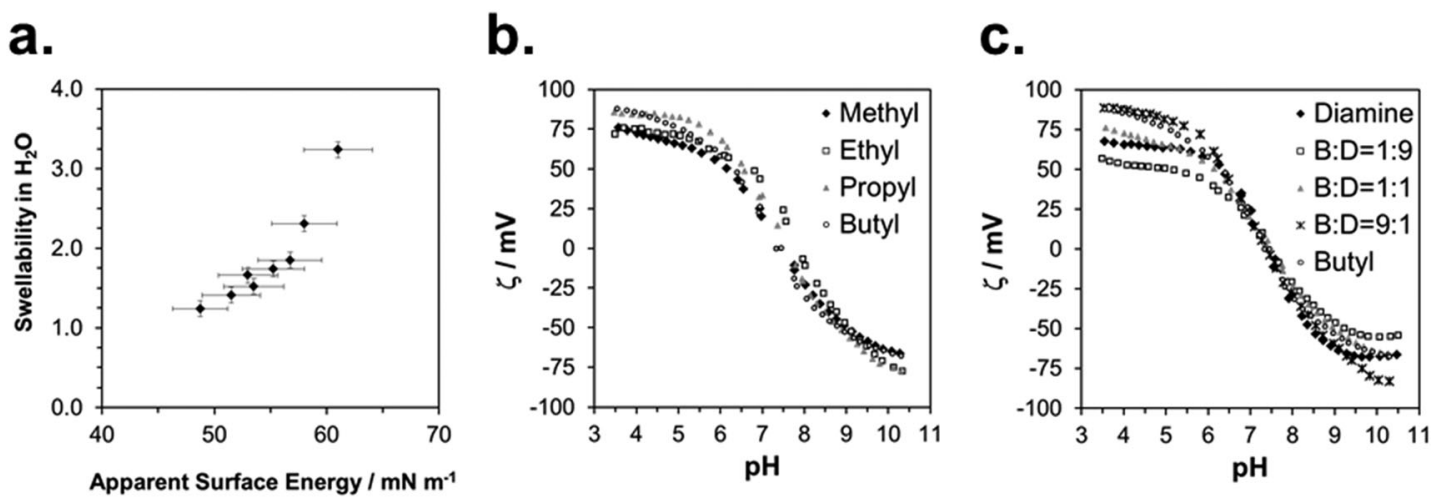

Fig. 4 Physical characterization of the SMAMP networks: (a) correlation between swellability in water and apparent surface energy; (b) representative $\zeta$ potential titration curves of the SMAMP homopolymer networks; (c) representative $\zeta$ potential titration curves of the butyl SMAMP copolymer networks.

solvents used. In our case, the polymer surfaces did swell, but slowly (>30 min). We therefore determined the static contact angle quickly after a defined time interval $(1 \mathrm{~min})$. This is a non-equilibrium measurement which only gives relative data; we therefore called these results apparent surface energies. The Zisman plots are given in the ESI $\dagger$ (page S20, Fig. S13-S15), the resulting apparent surface energies are included in Table 1.

To check the validity of these measurements, we plotted the swelling ratio in water versus the apparent surface energy (Fig. 4a). This gave an almost linear correlation. The only data point that significantly deviated from linearity was the one corresponding to the highly swollen diamine network. Thus, despite potential limitations of the Zisman method, apparent surface energies and swelling ratios in water were proportional, and therefore either parameter can be used to describe SMAMP hydrophobicity.

We measured the surface charge of the SMAMP networks using elektrokinetic measurements. ${ }^{* 24}$ During these measurements, the samples were titrated in situ from $\mathrm{pH} 2.5$ to $\mathrm{pH} 10.5$. The titration curves of $\zeta v$ s. $\mathrm{pH}$ (Fig. $4 \mathrm{~b}$ and c, respectively) conformed to the typical behavior of an amphiphilic polymer surface. At low $\mathrm{pH}$, the zeta potential had a positive plateau and went through a point of inflection. It then leveled off into a negative plateau at high pH. In Fig. $4 \mathrm{~b}$, the titration curves for the homopolymers with a hydrophobicity gradient from $\mathrm{R}=$ methyl to butyl are shown. The positive plateau values $\zeta_{\max }$ range from about $85 \mathrm{mV}$ for propyl and butyl, to about $75 \mathrm{mV}$ for methyl and ethyl, the two more hydrophilic polymers. We fitted this data using the Hill equation, a standard fit for sigmoidal curve shapes. $\dagger \dagger$ We also determined the isoelectric

** In the set-up used (SurPass, AntonPaar, Graz), the zeta potential $\zeta$ was obtained by measuring the streaming current through a sample chamber holding two planar, parallel sample slides. $\zeta$ was calculated from the Helmholtz-Smoluchowski equation $\zeta=\frac{\mathrm{d} I_{\text {str }}}{\mathrm{d} \Delta p} \cdot \frac{\eta}{\varepsilon \cdot \varepsilon_{0}} \cdot \frac{L}{A}$, where $\mathrm{d} I_{\text {str }}$ is the streaming current and $\Delta p$ the pressure difference during the measurement; $\eta, \varepsilon$ and $\varepsilon_{0}$ are parameters that describe the measurement electrolyte (viscosity, relative and absolute dielectric constant); $L$ and $A$ describe the sample geometry (distance between the sample slides and sample area, respectively). $\dagger \dagger=\zeta_{\max }+\left(\zeta_{\min }-\zeta_{\max }\right) \frac{\mathrm{pH}^{n}}{k^{n}+\mathrm{pH}^{n}}$. The fitting parameters were: $\zeta_{\max }$, the extrapolated start value of the fitted positive plateau; $\zeta_{\min }$, the extrapolated end value of the fitted negative plateau; $k$, the point of inflection and $n$, a measure of the width and steepness of the sigmoidal curve. point (IEP) and the acid constant ( $\mathrm{p} K$ value) of the surface bound acid-base pair of the polymer. The latter was calculated from $\mathrm{p} K=\mathrm{pH}\left(\frac{1}{2} \zeta_{\max }\right)+0.4343 \frac{F \zeta_{\max }}{2 R T}+\ddagger^{25}$ The data obtained is summarized in Table 1. The isoelectric points and $\mathrm{p} K$ values of the homopolymer networks differed about $0.5 \mathrm{pH}$ units, from 7.3 to 7.9 and 7.2 to 7.7 , respectively. The titration curves of the butyl copolymer series is shown in Fig. 4c. Here, the differences in $\zeta_{\max }$ ranged from 50 to $88 \mathrm{mV}$. Surprisingly, the polymers with higher nominal charge (more diamine content per unit length) had the lowest $\zeta_{\max }$ values. The reasons for this will be discussed below. The isoelectric points and $\mathrm{p} K$ values of the copolymers were much closer than in the homopolymer series, differing only by $0.2 \mathrm{pH}$ units.

The exact contribution of each chemical feature of a complicated polymer coating to the zeta potential is not yet fully understood, in particular if the sample can swell. It is clear that the acidbase pair $\mathrm{NH}_{3}{ }^{+} / \mathrm{NH}_{2}$ plays a significant role, as does the adsorption of electrolyte ions and aqueous counter-ions. ${ }^{24-26}$ Swelling influences the zeta potential significantly, and is typically observed by an additional minimum in the negative plateau at alkaline $\mathrm{pH} . \S \S \mathrm{We}$ used the Hill equation to calculate $\zeta$ under pseudo-physiological conditions by setting $\mathrm{pH}=7.4$. $\uparrow$ ศ Thus, we obtained apparent data which we could use to compare the surface properties at $\mathrm{pH}$ 7.4. It should also be mentioned that it was difficult to get consistent zeta potential data due to edge effects on the sample slides originating from the coating process. The Helmholtz-Smoluchowski equation relates streaming current and zeta potential. It contains the geometric factor $\frac{L}{A}$ (which depends on surface area $A$ ) and is

$\$ \neq \mathrm{pH}\left(\frac{1}{2} \zeta_{\max }\right)$ is the $\mathrm{pH}$ value where the half maximum of $\zeta$ is measured. The second term (containing Faraday's constant, the universal gas constant and the absolute temperature) is a term that corrects for the ionic strength.

$\S \S$ For $\mathrm{B}: \mathrm{D}=1: 9$ and the diamine polymer, the most strongly swelling polymers in this sample set, the titration curves at high $\mathrm{pH}$ bent slightly upwards, indicating that swelling effects were present.

Tा This is not exactly correct, because $\zeta$ is sensitive to ionic strength and was measured at $0.001 \mathrm{~mol} \mathrm{~L}^{-1}$ in $\mathrm{KCl}\left(=0.002 \mathrm{osmol} \mathrm{L}^{-1}\right)$, and not under physiological conditions of about $0.3 \mathrm{osmol} \mathrm{L}^{-1}$. However, this high salt concentration could not be used because it is outside the range in which the $\zeta$ can be measured with our set-up. $\zeta$ typically decreases with increasing ionic strength. 
thus sensitive to such effects. Still, several semi-quantitative observations could be derived, which can be used as a guideline to understand the biological effects. This is further discussed below.

The morphology of the SMAMP networks was characterized using atomic force microscopy (AFM). Representative AFM height and phase images are given in the ESI $\dagger$ (page S21, Tables S13 and S14). It is evident from these pictures that each SMAMP network homogeneously covered the wafer surface and only had distinct pores in the range of a few tens of nanometers. These originate from phase separation of the polymer and excess tetrathiol cross-linker. The depth of these pores was significantly less than the overall thickness of the network. We found previously that for the given network thickness of about $150 \mathrm{~nm}$, these pores do not impact the antimicrobial activity. ${ }^{27}$

\section{Antimicrobial activity of the SMAMP networks}

The antimicrobial activity of the SMAMP networks was determined using the so-called airborne antimicrobial assay. This assay simulates airborne bacterial contamination, e.g. through sneezing or coughing. We and others recently developed a standardized procedure for this assay. \|\|$^{27,28}$ Details are given in the ESI $\dagger$ (page S34). The results of the airborne assay are given in Fig. 5a-f. As this data shows, the SMAMP networks were highly efficient after two hours contact time - many of them killed close to $100 \%$ of the bacteria (Fig. $5 \mathrm{~d}-\mathrm{f}$ ). In particular, the $\mathrm{P}: \mathrm{D}=1: 9$, butyl, $\mathrm{B}: \mathrm{D}=9: 1, \mathrm{~B}: \mathrm{D}=5: 5$, and $\mathrm{B}: \mathrm{D}=1: 9$ had a reduction of at least $3 \log$ units (Fig. 5e and f). The most promising networks were $\mathrm{P}: \mathrm{D}=1: 9$ and $\mathrm{B}: \mathrm{D}=1: 9$, which quantitatively killed 3 out of 4 tested bacteria, and reduced the fourth by at least $3 \log$ units. While this is an exciting result from an applications point of view, it is not possible to correlate structural differences of the SMAMPs when the killing is nearquantitative. We therefore repeated the assay with only $30 \mathrm{~min}$ contact time to get a higher amount of surviving colony forming units (CFUs). This data is shown in Fig. 5a-c. For the Gram positive $S$. aureus bacteria, we observed a correlation between CFU reduction and increasing alkyl chain length in the homopolymer series (Fig. 5a). For Gram negative $E$. coli bacteria, there was an unexpected minimum for the ethyl networks, which had $100 \%$ killing efficiency (Fig. 5a). In the two copolymers series, there was a minimum in the CFU percentage (for $\mathrm{P}: \mathrm{D}=5: 5$ and $\mathrm{B}: \mathrm{D}=5: 5$, respectively) in the activities against Gram positive $S$. aureus and E. faecalis at $t=30 \mathrm{~min}$ (Fig. $5 \mathrm{~b}$ and c). This is also seen at $2 \mathrm{~h}$ contact time, where these two networks are the only ones in their series that quantitatively kill the two Gram positive bacteria (Fig. 5b and c). For the Gram negative E. coli and $P$. aeruginosa, the trend is less clear. At $30 \mathrm{~min}, E$. coli was reduced to about $0.5 \%$ remaining CFUs for propyl and all its copolymers. In the butyl copolymer series, the number of CFUs was one order of magnitude less, with a quantitative killing

|| || In short, bacteria are sprayed on a test surface and remain in contact with this surface for two hours. They are then removed and transferred onto agar plates. After incubation, the number of colony forming units (CFUs) is counted, and the antimicrobial activity is reported as the percentage of surviving CFUs (\%CFU) relative to a negative control. of $E$. coli for $\mathrm{B}: \mathrm{D}=9: 1$. P. aeruginosa had a minimum for $\mathrm{P}: \mathrm{D}=9: 1$ and $\mathrm{P}: \mathrm{D}=5: 5$ in the propyl series at $t=30 \mathrm{~min}$, and was killed quantitatively by all networks at $t=2 \mathrm{~h}$. In general, the Gram negative E. coli and $P$. aeruginosa were significantly more sensitive to the antimicrobial networks than the Gram positive S. aureus and E. faecalis, in many cases by two log units.

To correlate antimicrobial activity with physical properties, we plotted the percentage of surviving CFUs ( $\log$ (\%CFUs) for $E$. coli an $S$. aureus at $\left.t=30 \mathrm{~min},{ }^{* * *}\right)$ versus swellability, apparent surface energy, zeta potential, $\mathrm{p} K$ and the isoelectric point (Fig. 6a-f). The aim of these correlations was to differentiate between the effects of surface hydrophobicity and surface charge on antimicrobial activity. It is important to note that for most of the physical parameters we measured, the effect of changes in surface hydrophobicity and surface charge are coupled. For example, a higher surface charge will make a surface also more hydrophilic and cause more swelling. Likewise, a strong increase in hydrophobicity will also change the zeta potential. Thus, it is very difficult to experimentally separate the two parameters. The best parameter separation was achieved for the homopolymer series with $\mathrm{R}=$ methyl to butyl. Here, the nominal charge density per repeat unit was about the same, even in the swollen state, because the swelling ratios of these networks were not too different.

We compared these results to the butyl copolymer series with a simultaneous hydrophobicity and charge density gradient. For the homopolymers, we found a linear correlation in the $\log (\% \mathrm{CFUs})$ vs. swellability plot for both bacteria (Fig. 6a). For both copolymer series, there was a minimum in the $\log (\%$ CFUs) vs. swellability correlation for both bacteria (Fig. 6a). The same was found in the $\log (\%$ CFUs) vs. apparent surface energy plot (Fig. 6b): linear correlations were obtained for the homopolymers, minima for the copolymers.

The plots of parameters related to the zeta potential ( $\zeta_{\max }$, $\zeta_{\text {phys }}, \mathrm{p} K$ and IEP) vs. $\log (\% \mathrm{CFUs})$ are shown in Fig. 6c-f. Overall, these data points scatter more than the data in Fig. 6a and $b$, probably due to the mentioned edge effects. Still, interesting findings emerge: with few outliners, $\mathrm{p} K$ and IEP correlate with $\log (\% \mathrm{CFU})$ (Fig. 6e and f), no matter whether the data came from the homopolymer or copolymer series. Furthermore, the data for E. coli and S. aureus are well separated, indicating different sensitivities of these two bacteria to the polymer IEP and $\mathrm{p} K$. Thus, $\mathrm{p} K$ or IEP, at least in this series of structurally similar polymers samples, may be useful predictors for the antimicrobial activity of a surface against certain bacterial species. This is reasonable because the $\mathrm{p} K$ and IEP are sensitive to both the hydrophobic balance of the polymer and its charge density. In Fig. 6d, we also included a plot of the antimicrobial activity $v s$. the apparent physiological zeta potential. Even though the data scatters more widely, we also see a trend for this parameter.

\footnotetext{
*** For the other antimicrobial data sets, the percentage of CFUs was so low close to $100 \%$ killing - that we were in the limit of accuracy of the assay, where no meaningful correlations could be made.
} 


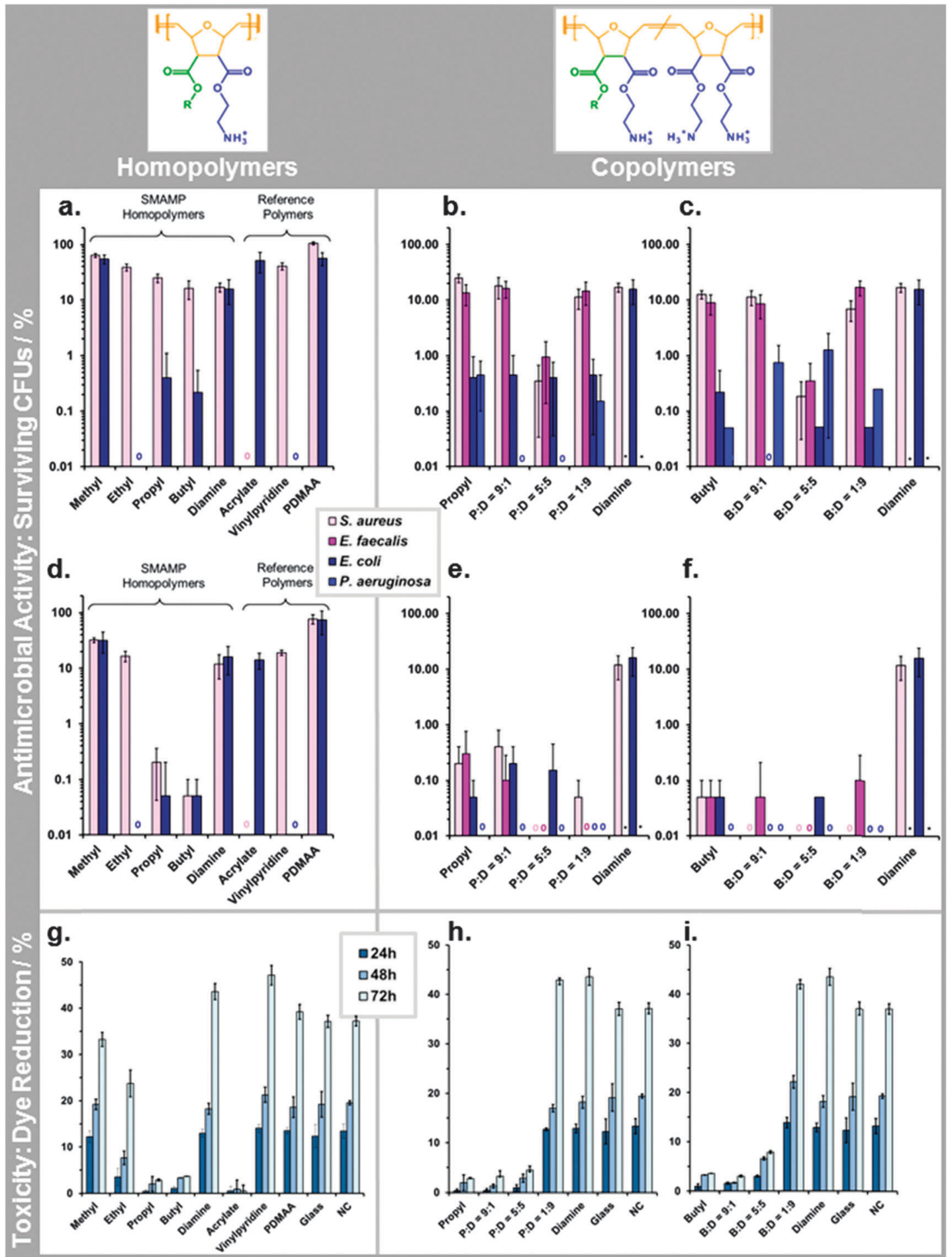

Fig. 5 Biological characterization of the SMAMP networks and the reference polymer networks (acrylate, vinylpyridine, PDMAA). (a-f) Antimicrobial activity (reported as percentage of surviving colony forming units, log(\%CFUs)) against various bacteria: (a) homopolymers, $t=30$ min; (b) and (c): propyl and butyl copolymers, $t=30 \mathrm{~min}$; (d) homopolymers, $t=2 \mathrm{~h}$; (e) and (f) propyl and butyl copolymers, $t=2 \mathrm{~h}$; (g-i) cell compatibility with primary human keratinocytes, reported as \% of Alamar Blue dye reduction after 24, 48 and $72 \mathrm{~h}$. (g) Homopolymers, (h) and (i) propyl and butyl copolymers.

\section{Cell compatibility of the SMAMP networks}

The cell toxicity of the polymer networks was quantified by two methods. First, primary human fibroblasts and immortalized human keratinocytes were grown on the SMAMP networks for
6 hours. Their RNA was then extracted and analyzed for up-regulation of toxicity markers using polymerase chain reaction (PCR). This data is shown in the ESI $\dagger$ (page S36, Fig. S24-S26). For the fibroblasts, the two apoptosis markers (ANXA5, CASP3), the inflammation marker IL6, and the cell proliferation marker 


\section{Hydrophobic Balance}

a.

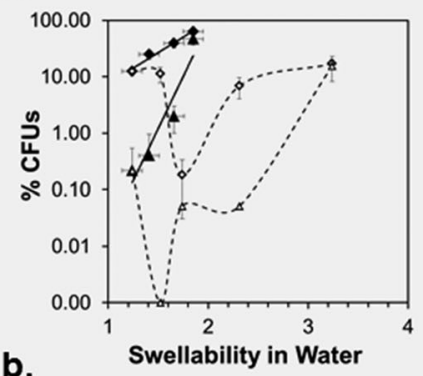

b.

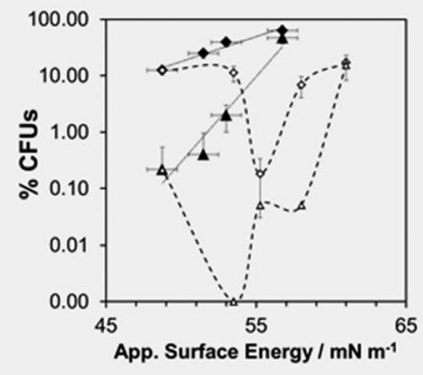

c.

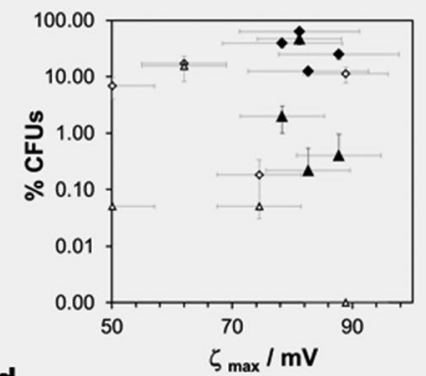

d.

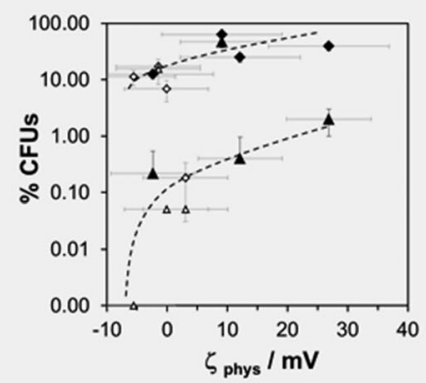

e.

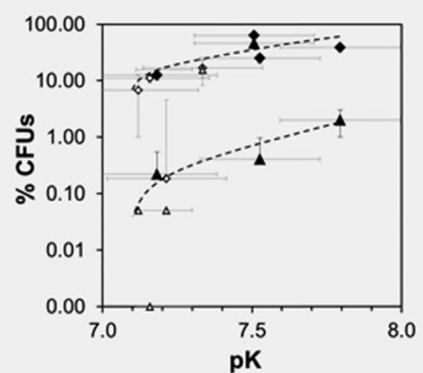

f.

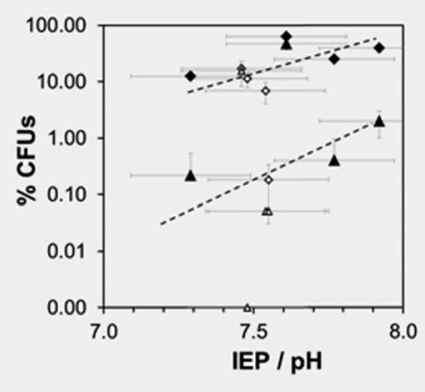

- Homopolymers, S. aureus, 30 min - Homopolymers, E. coli, 30 min
- Copolymers, S. aureus, 30 min

$\triangle$ Copolymers, E. coli, $30 \mathrm{~min}$

Fig. 6 Correlation of physical properties and antimicrobial activities of the surface-attached SMAMP networks: the percentage of surviving colony forming units, log\%CFUs, is plotted versus (a) swellability in water; (b) apparent surface energy; (c) maximum zeta potential; (d) zeta potential under pseudo-physiological conditions; (e) acid constant of the polymer network; and (f) isoelectric point of the polymer networks. All lines are guides to the eye only.

MKi67 were on the same level as the control for all SMAMPs (Fig. S25, ESI †). This is an indication that these networks were fully cell-compatible. For the keratinocytes, IL6 was significantly up-regulated (Fig. S26, ESI †), indicating an inflammatory response to the surface. To find out whether this was a significant sign for toxicity, we also performed the Alamar Blue assay on this cell type (ESI, $\dagger$ page 40). $\dagger \dagger \dagger$ The results are given in Fig. $5 g-i$. They nicely complement the PRC data. For the samples where IL6 was most up-regulated (butyl, propyl P:D $=9: 1$ and $\mathrm{P}: \mathrm{D}=5: 5$ ), Alamar Blue dye reduction was also low, indicating low cell viability. For the samples with low IL6 up-regulation $(\mathrm{B}: \mathrm{D}=1: 9$ and $\mathrm{P}: \mathrm{D}=1: 9$ ), the viability was close to that of the control, demonstrating good cell compatibility. For the homopolymer series, the cell toxicity increased with increasing alkyl chain length. The amount of chargeable units did not have an adverse effect on viability: the diamine homopolymer, $\mathrm{P}: \mathrm{D}=1: 9$ and $\mathrm{B}: \mathrm{D}=1: 9$ had an even higher dye reduction than the negative control, presumably because they swell strongly. For both copolymer series, cell toxicity was systematically reduced as hydrophobicity decreased.

$+\dagger$ In this assay, keratinocytes were cultivated on the SMAMP networks in the presence of Alamar Blue, a dye that is metabolized by viable cells. Thus, the amount of dye reduction relative to a negative control can be used to quantify cell viability.
We correlated these cell compatibility results with the physical properties of the surfaces by plotting the percentage of Alamar Blue dye reduction versus swellability, apparent surface energy, and parameters related to the zeta potential (Fig. 7). The $24 \mathrm{~h}$ and the $48 \mathrm{~h}$ time-point of the cell compatibility data were the most useful ones for these correlations, since the cells had substantially recovered from toxicological effects at $t=72$ hours. When plotting $\log (\%$ Dye reduction) vs. swellability (Fig. 7a), we observed a steep increase of the curve for low swellability, which then leveled off into a plateau. Thus, when a swellability ratio of about 2 was reached, making the surface more hydrophilic did not further improve the cell compatibility. Both copolymers and homopolymers conform to this curve. There is an inverse trend for the plot of dye reduction $v s$. $\zeta_{\max }$, where the amount of dye reduction decreases with increasing surface charge. The other surface charge parameters do not correlate with the cell compatibility data. This will be discussed below.

\section{Possible applications - coating of a model catheter}

To obtain proof-of-concept for a real-life application of our surface-attached SMAMP networks, we also coated medicalgrade PDMS tubing with propyl SMAMP networks. Since the standard process of network formation compromised the mechanical integrity of the tubing, we modified the process and developed parameters to directly apply the activated SMAMP coating to the 
Hydrophobic Balance

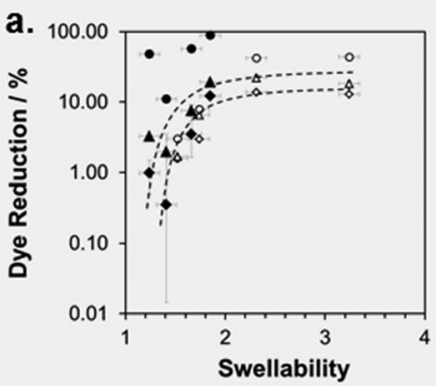

b.

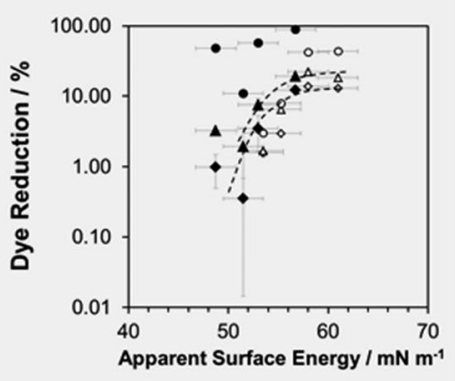

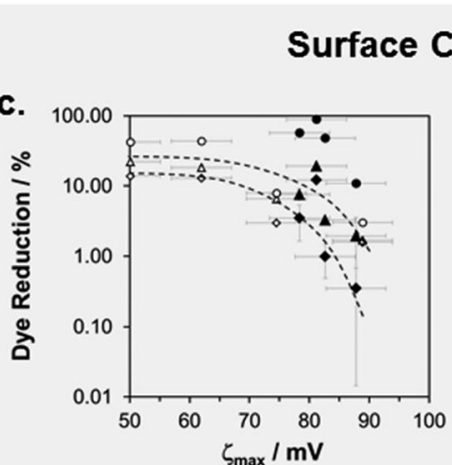

d.

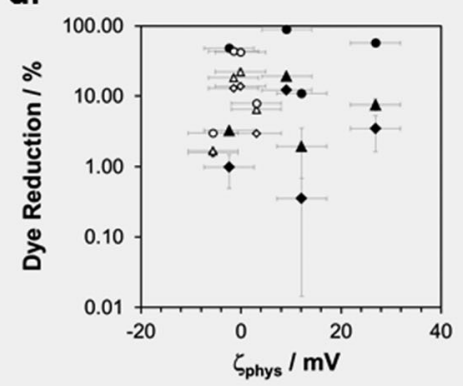

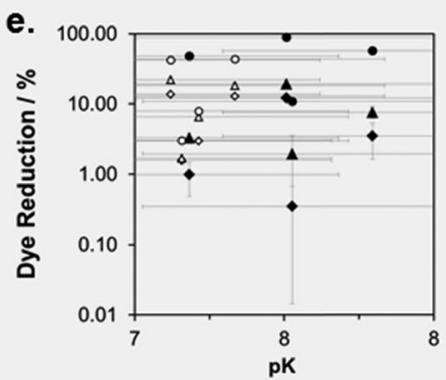

f.

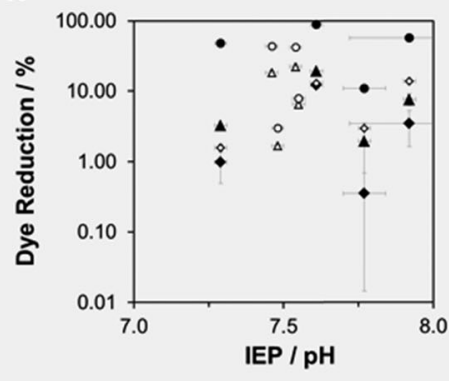

- Homopolymers, $24 \mathrm{~h}$

$\Delta$ Homopolymers, $48 \mathrm{~h}$

- Homopolymers, $72 \mathrm{~h}$
- Copolymers, $24 \mathrm{~h}$

$\triangle$ Copolymers, $48 \mathrm{~h}$

- Copolymers, $72 \mathrm{~h}$

Fig. 7 Correlation of physical properties and cell compatibility of surface-attached SMAMP networks: the percentage of Alamar Blue dye reduction is plotted versus (a) swellability in water; (b) apparent surface energy; (c) maximum zeta potential; (d) zeta potential under pseudo-physiological conditions; (e) acid constant of the polymer network; and (f) isoelectric point of the polymer networks.

PDMS tubing (ESI, $\dagger$ pages S27-S29). Two coatings of $100 \mathrm{~nm}$ thickness each were necessary to obtain an antibacterial efficacy of $>99.9 \%$ (Fig. S16, ESI $\dagger$ ). With this new process, which avoids corrosive chemicals and aggressive solvents in direct contact with the tubing, fully bactericidal SMAMP coatings on PDMS tubing were obtained.

\section{Discussion}

\section{Optimization of biological performance}

The first aim of the study was to use the modular chemistry of poly(oxonorbornenes) SMAMPs to obtain surface-attached polymer networks with systematically varying chemical structure. This allowed us to identify the SMAMP surface that had optimal broad band activity against Gram negative and Gram positive bacteria, and excellent compatibility towards human cells. These are crucial properties for all applications that are in contact with the human body for an extended period of time. By gradual variation of hydrophobicity and charge density, we found that $\mathrm{P}: \mathrm{D}=1: 9$, butyl, $\mathrm{B}: \mathrm{D}=9: 1, \mathrm{~B}: \mathrm{D}=5: 5$, and $\mathrm{B}: \mathrm{D}=1: 9$ had excellent antimicrobial properties. Out of these, $\mathrm{P}: \mathrm{D}=1: 9$ and $\mathrm{B}: \mathrm{D}=1: 9$ also had outstanding cell compatibility, while the others were more toxic. We had also synthesized antimicrobial networks based on the poly(methacrylates) reported by Kuroda and DeGrado, ${ }^{29}$ and the poly(vinylpyridines) by Tiller and Klibanov as a benchmark for our SMAMP networks. ${ }^{18,30}$ The data for these samples (included in Fig. 5a and d) showed that the acrylate only killed $S$. aureus, while the vinylpyridine was only active against $E$. coli. In the Alamar Blue assay, the acrylate was highly toxic, while the vinylpyridine had excellent cell compatibility. We further tested a surface-attached network made from poly(dimethylacrylamide) (PDMAA), ${ }^{22}$ which is known for its excellent cell compatibility, ${ }^{31}$ but is not antimicrobial (Fig. 5a and d). Comparing these three references to our SMAMPs, we find that the vinylpyridine was very similar to the ethyl homopolymer network. Further, none of the SMAMP networks was as toxic as the acrylate. $\mathrm{P}: \mathrm{D}=1: 9$ and $\mathrm{B}: \mathrm{D}=1: 9$, our most active SMAMPs, were as cell-friendly as PDMAA (Fig. 5g-i), but much more active. In the light of this data, we conclude that the $\mathrm{P}: \mathrm{D}=1: 9$ and $\mathrm{B}: \mathrm{D}=1: 9$ copolymers, with broadband antimicrobial activity and excellent cell compatibility, are meeting all the requirements for biomedical applications, and may be even setting a new standard for the field.

\section{Correlation of physical properties and antimicrobial activity}

The second lead question of our study was - how do the variations in chemical structure correlate with measurable physical quantities, and can we relate these to biological activity? In literature, structural changes in hydrophobicity (e.g. length of alkyl chain) or charge density (e.g. number of charged repeat units) had been previously compared with antimicrobial properties, but 
the effort typically remained on a qualitative level; physical parameters like zeta potential or surface energy were rarely measured. ${ }^{32}$ For the series of networks with constant charge density, our results show that swellability and apparent surface energy correlate linearly with antimicrobial activity- the higher the hydrophobicity, the higher the antimicrobial activity. For the series of networks where charge density is not constant, the situation is more complicated. Here, swellability and surface energy do not display any systematic correlation with antimicrobial activity. Thus, swellability and surface energy alone do not sufficiently to describe these systems. On the other hand, we found that $\mathrm{p} K$ and IEP correlated systematically with antimicrobial activity for all polymer networks. When scanning the literature for similar findings, we found only scarce reports on $\zeta$ measurements of antimicrobial polymer surfaces. ${ }^{32,33}$ In particular, pH-dependent measurements were rarely performed. Thus, our result that $\mathrm{p} K$ and IEP quantitatively correlated with antimicrobial activity, even when correlations to $\zeta_{\max }$ are not clear (which was the case for our strongly swelling samples) is an important extension to the previously found qualitative correlations between $\zeta_{\max }$ and antimicrobial activity. ${ }^{33 e_{2} f}$

How can we explain these findings? SMAMP networks are weak polyelectrolyte networks - their theoretical description is complicated, and surface-attached polyelectrolyte networks in particular are not yet fully understood. For example, it is not yet possible to predict the net surface charge of surface-attached weak polyelectrolyte networks. However, the following pieces of theory are useful to understand their behavior: when an unconstrained polyelectrolyte network swells, its chains are extended in three dimensions until there is equilibrium between the osmotic pressure inside the network and the surrounding solvent. Under these conditions, the swelling pressure $\Pi$ that drives solvent into the network is zero. ${ }^{34}$ In terms of free energy, $\Delta G=\Delta G_{\text {mix }}+\Delta G_{\text {elastic }}+\Delta G_{\text {ion }}=0$. This equation contains three terms. The one for the contribution of polymer-solvent mixing, $\Delta G_{\text {mix }}$, can be described by the Flory-Huggins-theory. ${ }^{35}$ The elastic energy $\Delta G_{\text {elastic }}$ is caused by stretching of the polymer chains out of their equilibrium conformation, and $\Delta G_{\text {ion }}$ describes the repulsive electrostatic interactions of the charged polyelectrolyte. ${ }^{34}$ Thus, $\Delta G_{\text {mix }}$ and $\Delta G_{\text {ion }}$ cause network extension, while $\Delta G_{\text {elastic }}$ is the restoring energy that limits swelling. This equation is also valid for surface-attached polyelectrolyte networks, with the exception that these can only swell normal to the surface. ${ }^{22}$ The problem to theoretically describe swollen surface-attached polyelectrolyte networks is that $\Delta G_{\text {ion }}$ and $\Delta G_{\text {elastic }}$ become mutually dependent through counter-ion condensation. ${ }^{36}$ The number of actual charges per unit volume in a polyelectrolyte network will depend on two factors - the distance between available chargeable units $d$ (which is dictated by the molecular structure) and the Bjerrum length $l .+\$$ Swelling increases $d$, and that may change the extent of counter-ion condensation if $d \leq l$.

For our surface-attached SMAMP homopolymer network series, with a constant number of chargeable sites per unit

\$t The Bjerrum length is the distance where the thermal energy of two charges is equal to their electrostatic repulsion, i.e. the minimum distance of two charges. volume, we estimate that $d$ is on the order of magnitude of $0.75 \mathrm{~nm}$ (about 5 carbon-carbon bonds between each $\mathrm{NH}_{3}{ }^{+}$ group). This compares to $l=0.7 \mathrm{~nm}$ in water at room temperature. Since SMAMPs are weak polyelectrolytes, we have to consider the acid-base equilibrium of the $\mathrm{NH}_{3}{ }^{+} / \mathrm{NH}_{2}$ acid-base pair. With a $\mathrm{p} K$ of 7.3-7.9 in the homopolymer series, these groups dissociate $50 \%$ or less under physiological conditions $(\mathrm{pH}=7.4)$. Consequently, $d>l$ even without swelling effects. Thus, within this series, the actual surface charge of the homopolymers is dominated by the acid-base equilibrium and not by counter ion condensation, and $\Delta G_{\text {ion }}$ is roughly constant for all homopolymer networks. This is consistent with a difference in $\zeta_{\max }$ of only $11 \mathrm{mV}$ for the homopolymer series (Fig. $4 \mathrm{~b}$ and Table 1). Under these conditions, changes in $\Delta G_{\text {mix }}$ and $\Delta G_{\text {elastic }}$ through the homopolymer series must compensate each other. Swelling only occurs if $\Delta G_{\text {mix }}$ is negative. The modulus of $\Delta G_{\text {mix }}$ should be larger for the more hydrophilic networks, $\left|\Delta G_{\text {mix,methyl }}\right|>\left|\Delta G_{\text {mix,ethyl }}\right|>\left|\Delta G_{\text {mix,Proyl }}\right|>$ $\left|\Delta G_{\text {mix,butyl }}\right|$. Consequently, for the absolute values of the elastic energy stored in the swollen networks, $\Delta G_{\text {elastic,methyl }}>$ $\Delta G_{\text {elastic,ethyl }}>\Delta G_{\text {elastic,Proyl }}>\Delta G_{\text {elastic,butyl }}$. This is consistent with the experimentally found decreasing swelling ratio of 1.9 to 1.2 from methyl to butyl. Thus, what we see when we measure swellability of the SMAMP homopolymer networks are differences in $\Delta G_{\text {mix }}$, and consequently in the Flory-Hugginsparameter $\chi$ of these polymers. Therefore, for these equally charged, equally cross-linked, equally thick SMAMP networks, $\chi$ correlates with antimicrobial activity.

In contrast to the above discussed homopolymers, our copolymers have two chargeable amine groups per repeat unit, and a distance $d$ of about $0.4 \mathrm{~nm}$. Additionally, they have a slightly lower $\mathrm{p} K(7.2)$ and are thus slightly more dissociated $(60 \%$ at $\mathrm{pH}$ 7.4). Therefore, for the non-swollen copolymer networks, $d \leq l$, and counter-ion condensation becomes significant for the series members with high diamine content. When these networks swell, the extent of counter-ion condensation changes, and we are crossing into the regime where $d \geq e$. Thus, swelling affects both $\Delta G_{\text {elastic }}$ and $\Delta G_{\text {ion }}$. Experimentally, we observe a systematic increase of swelling with increasing diamine content. This is consistent with an increase of $\left|\Delta G_{\text {mix }}\right|$, and also with an increasing absolute value of $\Delta G_{\text {elastic. }}$ The question is - how exactly is $\Delta G_{\text {ion }}$ affected? This should be mirrored in $\zeta_{\max }$. We do not see a systematic trend for $\zeta_{\max }$, which in fact goes through a minimum for $\mathrm{B}: \mathrm{D}=1: 9$ and is smaller for diamine than for butyl, because diamine swells much more strongly. This non-linear behavior, and the resulting non-linear behavior of antimicrobial activity in the copolymer series, may be explained by the overlay of the steadily increasing parameter $\Delta G_{\text {mix }}$, which causes swelling, and the non-linear changes in the extent of counter-ion condensation, which affect $\Delta G_{\text {ion }}$.

In the absence of a more quantitative theory for surfaceattached weak polyelectrolyte networks, further correlations are difficult. It is very interesting however, that the parameter $\mathrm{p} K$ obtained from the $\zeta$ potential titrations correlates with antimicrobial activity for all polymers that were tested. Since the $\mathrm{p} K$ data for the homopolymer series shows that at equal 
a.

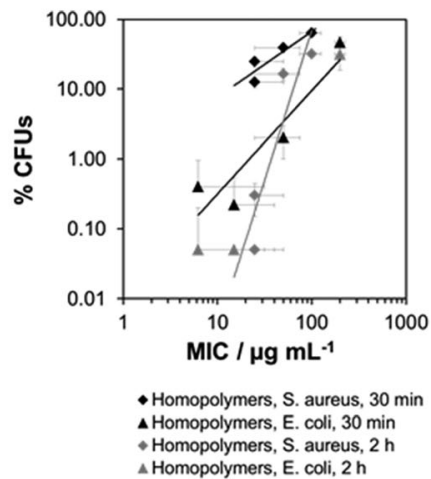

b.

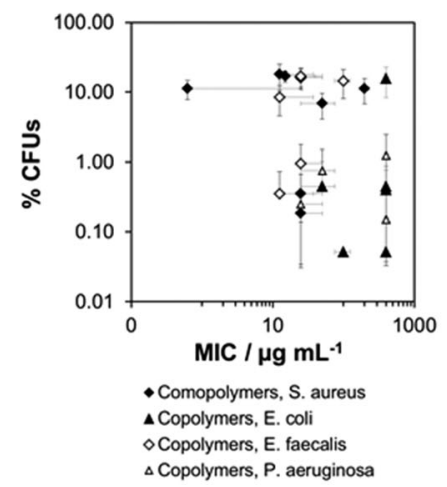

c.

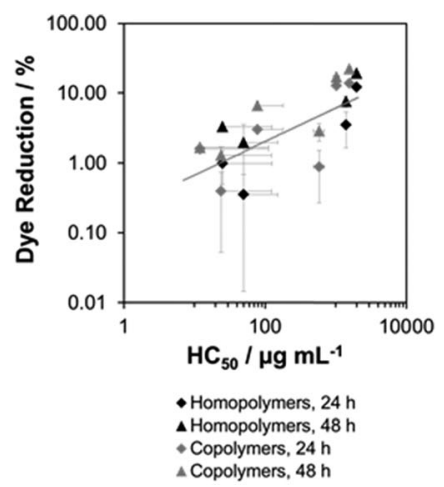

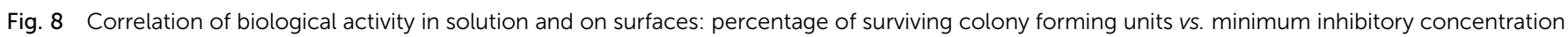

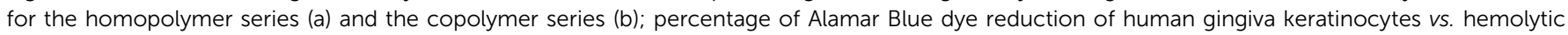
concentration (human red blood cells, c).

charge density, $\mathrm{p} K$ changes with hydrophobicity, we find that it is a parameter that is not only affected by the acid-base equilibrium, but also by the overall hydrophobicity of the surface-attached network. This is also well-known for proteins. $\mathrm{p} K$ may therefore be the most sensitive parameter in our data set that takes into account both effects - charge and hydrophobicity changes - and could be a useful predictor for antimicrobial activity in a given polymer series. It should be noted however that $\mathrm{p} K$ primarily depends on the chemical nature of the cationic group. Thus we can explain a shift in $\mathrm{p} K$ for structurally similar polymers, but would not yet dare to propose a general correlation of $\mathrm{p} K$ with antimicrobial activity for different cations.

\section{Correlation of physical properties and cell compatibility}

We observed an unambiguous correlation between swellability and cell compatibility for all homopolymer and copolymer networks (Fig. 7a). The curve of $\log (\%$ Dye reduction) $v s$. swellability first had a steep slope and then leveled into a plateau. Since simultaneous antimicrobial activity and cell compatibility is a subtle balance, the plateau in this curve is an important finding, as it gives a design rule to the polymer chemist. The optimal coating will be found slightly to the left of the plateau. Since swellability and apparent surface energy correlate (Fig. 4a), the same trend is found in the $\log$ (Dye reduction) vs. apparent surface energy plot (Fig. 7b). Thus, swellability and apparent surface energy are both suitable to optimize coatings for biocompatibility by physical methods. We found a similar curve shape in the $\log$ (Dye reduction) vs. $\zeta_{\max }$ plots (Fig. 7c). This is consistent with literature reports on the toxicity of too highly charged cationic surfaces.

\section{Correlation of biological activity on surfaces and in solution}

Lastly, we wanted to see if it was possible to quantitatively correlate the biological activity of SMAMPs on surfaces and in solution. The solution properties of some of our SMAMPs, particularly antimicrobial activity and hemolytic activity, have been carefully characterized before. ${ }^{7 b, d, 9 a}$ We plotted the antimicrobial data of the here reported SMAMP homopolymer networks (for $S$. aureus and E. coli, respectively) vs. the MIC§§§ data for these homopolymers in solution (at a molecular weight of $3000 \mathrm{~g} \mathrm{~mol}^{-1}$, data from ${ }^{7 d}$ ). This is shown in Fig. 8a. The number of available data-points was scarce, nevertheless there seems to be a positive correlation for S. aureus and E. coli - the higher the solution activity, the higher the surface activity (Fig. 8a). For the copolymers, with simultaneous changes in charge density and hydrophobicity, no such correlation could be found (Fig. 8b).

To correlate the cell compatibility of SMAMPs on surfaces and in solution, we plotted the amount of Alamar Blue dye reduction on surfaces $v s$. the hemolytic concentration in solution. Although the data also scatters, there is a clear correlation in the $\log (\%$ Dye reduction $) v s$. $\log \left(\mathrm{HC}_{50}\right)$ बा plot for homopolymers and copolymers. This indicates that the same parameters govern cell compatibility on surfaces and in solution, and that $\mathrm{HC}_{50}$ is a useful method to also predict the biocompatibility of surface-attached polymers.

\section{Mechanistic considerations}

While the mechanism of action of natural antimicrobial peptides (AMPs) and of antimicrobial polymers in solution is meanwhile partially understood, ${ }^{37}$ the mechanism by which surfaceattached polymers kill pathogens is still under debate. Early papers describe a 'hole-poking mechanism', ${ }^{11 a, d, 38}$ which is probably more of a cell membrane penetration and perturbation by not too densely grafted, surface-attached polyelectrolyte brushes, since such brushes are collapsed and not spike-like under physiological, high salt conditions. The impact of charge density on antimicrobial activity was discussed by Ober, ${ }^{39}$ Russell and Matyjaszewski, ${ }^{10 a, b}$ and Kügler et al., who even found a species-dependent charge threshold for activity. ${ }^{10 c}$ As to mechanisms, it was suggested that the exchange of the divalent

$\S \S \S$ The MIC (= minimum inhibitory concentration) is the smallest concentration that inhibits bacterial growth.

ฯศ The $\mathrm{HC}_{50}$ (= hemolytic concentration) is the concentration where $50 \%$ of red blood cells were damaged. 


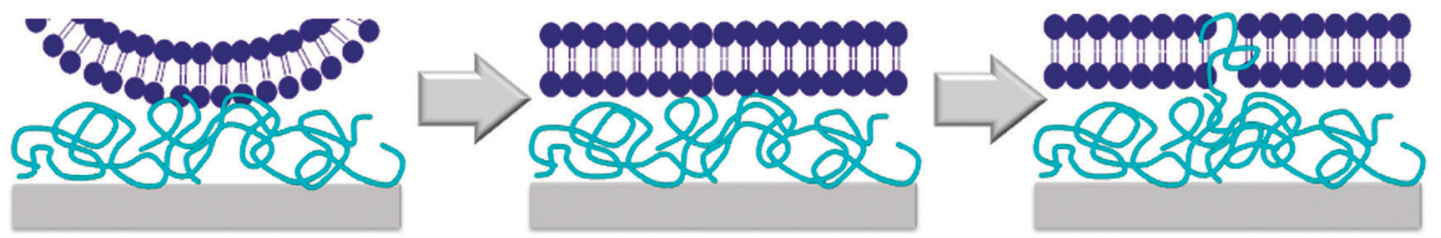

Fig. 9 Cartoon illustration of the modified mechanism of action of cationic antimicrobial surfaces: first, the bacterially are locally flattened by the high charge density of the surface. This enables interaction with the polymer segments on the surface, which insert into the membrane and cause damage that may lead to cell death.

membrane counter-ions against the polyelectrolyte surface was lethal to bacteria. ${ }^{10 c, 40}$ In a recent paper, Chan-Park suggests that highly charged surfaces can suck anionic components out of cell membranes, like an 'anion sponge'. ${ }^{15}$ Tiller independently also proposed such a mechanism ('lipid sponge effect'). ${ }^{16}$ Busscher recently postulated that there is a 'lethal regime', in which the adhesion between bacteria and the cationic substrate is so strong that bacteria are unable to detach and grow, and eventually die. ${ }^{17,41}$ The existence of such a charge-dependent lethal regime would explain Kügler's species-dependent charge thresholds ${ }^{10 c}$ and match the observations of Tiller and Busscher, ${ }^{17}$ Chan-Park, ${ }^{15}$ Matyjaszewski and Russell. ${ }^{10 a, b}$ We also found a species-dependent correlation between charge and antimicrobial activity (Fig. 6d-f).

Such a charge based-mechanism alone, however, does not explain all the observations in this work. It does not explain differences in antimicrobial activity of equally charged polymers with different hydrophobicity, which was observed in our welldefined model system (Fig. 6a and b), and also reported by others. ${ }^{42}$ Charge density therefore cannot be the whole answer to explain antimicrobial activity on surfaces - the role of hydrophobicity needs to be included into any proposed mechanism. Busscher suggested that the mechanism of action of antimicrobial polymers in solution may be fundamentally different to the mechanism of action of antimicrobial polymers on surfaces. ${ }^{17}$ In contrast to this, we observed a correlation of antimicrobial activity in solution and on surface for equally charged polymers (Fig. 8a). This would be impossible if the two mechanisms were fundamentally different in all aspects. However, we see no such correlation when we compare the solution and surface activity of polymers with a simultaneous hydrophobicity and charge gradient (Fig. 8b). To understand this, let us look at the mechanism of action of antimicrobial peptides and polymers in solution. The currently accepted model is a two-stage process. Long range electrostatic forces first enable attachment of the cationic antimicrobial molecules to the bacterial surface. In the second step, the hydrophobic groups interact with the bacterial membrane and cause disruption or destabilization. To us, the here reported findings are a strong indication that the mechanism of contact-killing antimicrobial surfaces is also a multi-step process. The step dominated by hydrophobicity $\left(\Delta G_{\text {mix }}\right)$ should be similar to the one found in solution: once in contact with the bacterial surface, the flexible segments of the antimicrobial polymer insert into the bacterial membrane and cause disruption. Thus, the higher the hydrophobicity at constant charge density, the higher the antimicrobial activity. Such an insertion is possible even for surface-attached polymers because they contain swollen, flexible chains or chain segments, and are not rigid bodies. The other mechanistic feature must be chargedominated, as described by Busscher, ${ }^{17}$ Tiller $^{16}$ and Chan-Park. ${ }^{15}$ The body of data from literature seems to indicate that, unlike in solution, the role of charge is not just to attract the antimicrobial species to the bacterial membrane, and then let hydrophobicity operate. Because the charge density near a cationic surface is much higher than the charge density of low molecular weight antimicrobial polymers in solution, the cationic surface also impacts the viability of bacteria, which cannot fulfil vital functions because they are pinned to the surface by charge. The considerations can be summed up in the following mechanism cartoon (Fig. 9): first, bacteria that have approached the surface are flattened out in the contact zone due to the high local charge density of the antimicrobial surfaces. This flattening will enable close contact between the bacterial envelope and the surface, so that the polymer chain segments at the interface can insert into the bacterial membrane.

Depending on the relative changes in charge density, $\Delta\left(\Delta G_{\text {ion }}\right)$, or hydrophobicity, $\Delta\left(\Delta G_{\text {mix }}\right)$, within a polymer series, one mechanistic feature may dominate the trends in that series, and there may even be opposite effects. For our homopolymer series, we could clearly show the effects of the hydrophobicity-driven mode of action, but we could not yet demonstrate the workings of changes in charge density, because we could not yet design a system where charge changes without affecting hydrophobicity.

\section{Conclusion}

Antimicrobial polymer surfaces are attractive materials to fend off the propagation of bacterial pathogens. In this paper, we presented an easy method to obtain surface-attached networks made from poly(oxonorbornene)-based synthetic mimics of antimicrobial peptides (SMAMPs). We systematically varied the structural properties of these networks, and correlated them to physical and biological properties. We could show for surface-attached SMAMP networks with similar thickness and cross-linker density that

- by carefully balancing hydrophobicity and charge density, broad-band active antimicrobial surface-attached networks with excellent cell compatibility were obtained, which can also be used in real-life applications such as catheter coatings.

- at constant charge density, swellability and apparent surface energy decreased with increasing antimicrobial activity. This indicates a hydrophobicity-related step in the mechanism of action of antimicrobial polymer networks. 
- the acid constant $\mathrm{p} K$ correlates decreased with increasing antimicrobial activity for all polymer networks investigated, and thus may be a useful property to predict the relative antimicrobial activity of a series of structurally related polymer networks.

- swellability and apparent surface energy increased with increasing cell compatibility.

- cell compatibility decrease with increasing $\zeta_{\max }$. This is consistent with reports that highly charged surfaces are not cell-friendly.

- for equally charged surface-attached antimicrobial networks, antimicrobial activities on surfaces and in solution correlate. This is an indication that the hydrophobic component in the mechanism that describes antimicrobial action in solution and on surfaces is related.

- for networks where hydrophobicity and charge density vary simultaneously, no such correlation was found. This indicates that the step in the mechanism of action that is charge driven differs significantly in solution and on surfaces.

- for all networks, cell compatibility in solution and on surfaces correlate, indicating that the principle that governs these phenomena is similar in both cases.

To our knowledge, this is the first work where such extensive correlations become apparent. However, further studies are needed to derive equations that describe the relations between biological activity and the here investigated physical parameters. We also have to stress that so far, these correlations hold only for structurally closely related compounds. We invite other researchers to complement their studies with these types of measurements, so that a broader data basis for more universal correlations between physical parameters and biological is obtained, which would be of great benefit for the whole field.

Additionally, it has to be noted that the parameters measured here were determined on the macroscopic scale; however, bacteria and cells are micro-objects which may react very sensitive to local effects. For example, they respond to hydrophobic excess in a not-quite randomly-distributed polymer chain, ${ }^{43}$ or to nanometer-sized surface features. These are effects which may impact the biological activities so strongly that the above observed trends (which we obtained from structurally regular, well-defined, facially amphiphilic SMAMP networks) may not be observable in less homogeneous systems.

Finally, it is well known that cationic polymer surfaces tend to have reduced activity in vivo due to protein adsorption and contamination with biofilms. Thus, to be efficient in medical applications, antimicrobial activity alone is not sufficient. Rather, the ideal material for clinical applications should be also antifouling or self-cleaning. Some progress in this direction has been made: for example, it was shown that some surfaces can be regenerated easily by washing with a cationic surfactant, which is a promising step towards long-term sterile antimicrobial coatings. ${ }^{11 c}$ We also reported combined surfaces of antimicrobial SMAMPs and antifouling polyzwitterions that were protein-resistant. ${ }^{21}$ These are first steps towards long-term active surfaces that are dearly needed for hospital applications.

\section{Experimental}

All experimental procedures are given in the ESI. $\dagger$

\section{Acknowledgements}

Funding of this project by an Emmy-Noether Grant of the German Research Foundation (DFG, LI1714/5-1) to K.L. is gratefully acknowledged. The catheter coating part of the project has been funded by OphthaSwiss, Herisau. Jürgen Rühe is gratefully acknowledged for very helpful discussions and sharing of many analytical instruments. Experiments were conducted by P.Z., D.L., E.K.R., Z.D., F.D., H.-R.P.-H., and D.L.G.S. T.S. and A.A.-A. designed the biological experiments, K.L. designed and directed the project, evaluated the data for this manuscript and her habilitation thesis, made the figures, and wrote the manuscript. P.Z. compiled the data and made most of the figures for the ESI, $\dagger$ and independently evaluated the data for his doctoral thesis.

\section{References}

1 World Health Organization, Report on global surveillance of antimicrobial resistance, 2014.

2 (a) Center for Disease Control, http://www.cdc.gov/mrsa; (b) European Antimicrobial Resistance Surveillance Network (EARS-Net), Antimicrobial resistance surveillance in Europe 2013, http://www.ecdc.europe.eu/publications.

3 (a) O. A. Awoyinka, I. O. Balogun and A. A. Ogunnowo, J. Med. Plants Res., 2007, 1, 63-65; (b) K. Page, M. Wilson and I. P. Parkin, J. Mater. Chem., 2009, 19, 3819-3831.

4 (a) S. J. Dancer, J. Hosp. Infect., 2004, 56, 10-15; (b) B. Dietze, A. Rath, C. Wendt and H. Martiny, J. Hosp. Infect., 2001, 49, 255-261; (c) J. M. Boyce, G. Potter-Bynoe, C. Chenevert and T. King, Infect. Control Hosp. Epidemiol., 1997, 18, 622-627.

5 R. M. Donlan and J. W. Costerton, Clin. Microbiol. Rev., 2002, 15, 167-193.

6 (a) C. von Eiff, B. Jansen, W. Kohnen and K. Becker, Drugs, 2005, 65, 179-214; (b) E. M. Hetrick and M. H. Schoenfisch, Chem. Soc. Rev., 2006, 35, 780-789.

7 (a) K. Lienkamp and G. N. Tew, Chem. - Eur. J., 2009, 15, 11784-11800; (b) K. Lienkamp, A. E. Madkour, K.-N. Kumar, K. Nuesslein and G. N. Tew, Chem. - Eur. J., 2009, 15, 11715-11722; (c) K. Lienkamp, K.-N. Kumar, A. Som, K. Nuesslein and G. N. Tew, Chem. - Eur. J., 2009, 15, 11710-11714; (d) K. Lienkamp, A. E. Madkour, A. Musante, C. F. Nelson, K. Nusslein and G. N. Tew, J. Am. Chem. Soc., 2008, 130, 9836-9843.

8 (a) M. Zasloff, Nature, 2002, 415, 389-395; (b) K. A. Brogden, M. Ackermann, P. B. McCray and B. F. Tack, Int. J. Antimicrob. Agents, 2003, 22, 465-478; (c) K. A. Brogden, Nat. Rev. Microbiol., 2005, 3, 238-250.

9 (a) A. Al-Ahmad, D. Laird, P. Zou, P. Tomakidi, T. Steinberg and K. Lienkamp, PLoS One, 2013, 8, e73812; (b) A. E. Madkour, A. H. R. Koch, K. Lienkamp and G. N. Tew, Macromolecules, 2010, 43, 4557-4561.

10 (a) J. Y. Huang, R. R. Koepsel, H. Murata, W. Wu, S. B. Lee, T. Kowalewski, A. J. Russell and K. Matyjaszewski, Langmuir, 
2008, 24, 6785-6795; (b) H. Murata, R. R. Koepsel, K. Matyjaszewski and A. J. Russell, Biomaterials, 2007, 28, 4870-4879; (c) R. Kügler, O. Bouloussa and F. Rondelez, Microbiology, 2005, 151, 1341-1348.

11 (a) J. C. Tiller, C. J. Liao, K. Lewis and A. M. Klibanov, Proc. Natl. Acad. Sci. U. S. A., 2001, 98, 5981-5985; (b) J. C. Tiller, S. B. Lee, K. Lewis and A. M. Klibanov, Biotechnol. Bioeng., 2002, 79, 465-471; (c) J. Lin, J. C. Tiller, S. B. Lee, K. Lewis and A. M. Klibanov, Biotechnol. Lett., 2002, 24, 801-805; (d) J. Lin, S. Y. Qiu, K. Lewis and A. M. Klibanov, Biotechnol. Bioeng., 2003, 83, 168-172; (e) J. Lin, S. Y. Qiu, K. Lewis and A. M. Klibanov, Biotechnol. Prog., 2002, 18, 1082-1086; $(f)$ D. Park, J. Wang and A. M. Klibanov, Biotechnol. Prog., 2006, 22, 584-589; ( $g$ ) N. M. Milovic, J. Wang, K. Lewis and A. M. Klibanov, Biotechnol. Bioeng., 2005, 90, 715-722.

12 J. Thome, A. Hollander, W. Jaeger, I. Trick and C. Oehr, Surf. Coat. Technol., 2003, 174, 584-587.

13 A. E. Madkour, J. A. Dabkowski, K. Nusslein and G. N. Tew, Langmuir, 2009, 25, 1060-1067.

14 (a) S. B. Lee, R. R. Koepsel, S. W. Morley, K. Matyjaszewski, Y. J. Sun and A. J. Russell, Biomacromolecules, 2004, 5, 877-882; (b) J. Y. Huang, H. Murata, R. R. Koepsel, A. J. Russell and K. Matyjaszewski, Biomacromolecules, 2007, 8, 1396-1399.

15 P. Li, Y. F. Poon, W. Li, H.-Y. Zhu, S. H. Yeap, Y. Cao, X. Qi, C. Zhou, M. Lamrani, R. W. Beuerman, E.-T. Kang, Y. Mu, C. M. Li, M. W. Chang, S. S. J. Leong and M. B. Chan-Park, Nat. Mater., 2011, 10, 149-156.

16 A. M. Bieser and J. C. Tiller, Macromol. Biosci., 2011, 11, 526-534.

17 L. A. T. W. Asri, M. Crismaru, S. Roest, Y. Chen, O. Ivashenko, P. Rudolf, J. C. Tiller, H. C. van der Mei, T. J. A. Loontjens and H. J. Busscher, Adv. Funct. Mater., 2014, 24, 346-355.

18 J. C. Tiller, C.-J. Liao, K. Lewis and A. M. Klibanov, Proc. Natl. Acad. Sci. U. S. A., 2001, 98, 5981-5985.

19 K. Kuroda, G. A. Caputo and W. F. DeGrado, Chem. - Eur. J., 2009, 15, 1123-1133.

20 O. Prucker, C. A. Naumann, J. Rühe, W. Knoll and C. W. Frank, J. Am. Chem. Soc., 1999, 121, 8766-8770.

21 P. Zou, W. Hartleb and K. Lienkamp, J. Mater. Chem., 2012, 22, 19579-19589.

22 R. Toomey, D. Freidank and J. Rühe, Macromolecules, 2004, 37, 882-887.

23 R. J. Good, J. Adhes. Sci. Technol., 1992, 6, 1269-1302.

24 C. Werner, H. Körber, R. Zimmermann, S. Dukhin and H.-J. Jacobasch, J. Colloid Interface Sci., 1998, 208, 329-346.

25 H.-J. Jacobasch, Prog. Org. Coat., 1989, 17, 115-133.

26 (a) C. Werner, U. Konig, A. Augsburg, C. Arnhold, H. Korber, R. Zimmermann and H. J. Jacobasch, Colloids Surf., A, 1999, 159, 519-529; (b) H.-J. Jacobasch, F. Simon, C. Werner and C. Bellmann, Tech. Mess., 1996, 63, 447.

27 A. Al-Ahmad, P. Zou, D. L. Guevara-Solarte, E. Hellwig, T. Steinberg and K. Lienkamp, PLoS One, 2014, e111357.

28 J. Haldar, A. K. Weight and A. M. Klibanov, Nat. Protoc., 2007, 2, 2412-2417.
29 (a) E. F. Palermo, I. Sovadinova and K. Kuroda, Biomacromolecules, 2009, 10, 3098-3107; (b) K. Kuroda and W. F. DeGrado, J. Am. Chem. Soc., 2005, 127, 4128-4129.

30 J. C. Tiller, S. B. Lee, K. Lewis and A. M. Klibanov, Biotechnol. Bioeng., 2002, 79, 465-471.

31 C. K. Pandiyarajan, O. Prucker, B. Zieger and J. Rühe, Macromol. Biosci., 2013, 13, 873-884.

32 A. M. Kelly, V. Kaltenhauser, I. Muehlbacher, K. Rametsteiner, H. Kren, C. Slugovc, F. Stelzer and F. Wiesbrock, Macromol. Biosci., 2013, 13, 116-125.

33 (a) J. A. Lichter and M. F. Rubner, Langmuir, 2009, 25, 7686-7694; (b) B. Gottenbos, D. W. Grijpma, H. C. Van der Mei, J. Feijen and H. J. Busscher, J. Antimicrob. Chemother., 2001, 48, 7-13; (c) D. Iarikov Dmitri, M. Kargar, A. Sahari, L. Russel, T. Gause Katelyn, B. Behkam and A. Ducker William, Biomacromolecules, 2014, 15, 169-176; (d) S. Y. Wong, L. Han, K. Timachova, J. Veselinovic, M. N. Hyder, C. Ortiz, A. M. Klibanov and P. T. Hammond, Biomacromolecules, 2012, 13, 719-726; (e) G. Seyfriedsberger, K. Rametsteiner and W. Kern, Eur. Polym. J., 2006, 42, 3383-3389; $(f)$ M. L. Gupta, K. Brunson, A. Chakravorty, P. Kurt, J. C. Alvarez, F. Luna-Vera and K. J. Wynne, Langmuir, 2010, 26, 9032-9039. 34 H. H. Hooper, J. P. Baker, H. W. Blanch and J. M. Prausnitz, Macromolecules, 1990, 23, 1096-1104.

35 P. J. Flory, J. Chem. Phys., 1942, 10, 51-61.

36 G. S. Manning and J. Ray, J. Biomol. Struct. Dyn., 1998, 16, 461-476.

37 (a) E. A. Porter, X. F. Wang, H. S. Lee, B. Weisblum and S. H. Gellman, Nature, 2000, 404, 565; (b) D. H. Liu and W. F. DeGrado, J. Am. Chem. Soc., 2001, 123, 7553-7559; (c) T. L. Raguse, E. A. Porter, B. Weisblum and S. H. Gellman, J. Am. Chem. Soc., 2002, 124, 12774-12785; (d) G. N. Tew, D. H. Liu, B. Chen, R. J. Doerksen, J. Kaplan, P. J. Carroll, M. L. Klein and W. F. DeGrado, Proc. Natl. Acad. Sci. U. S. A., 2002, 99, 5110-5114; (e) J. A. Patch and A. E. Barron, Curr. Opin. Chem. Biol., 2002, 6, 872-877; $(f)$ D. H. Liu, S. Choi, B. Chen, R. J. Doerksen, D. J. Clements, J. D. Winkler, M. L. Klein and W. F. DeGrado, Angew. Chem., Int. Ed., 2004, 43, 1158-1162; $(g)$ K. Kuroda and W. F. DeGrado, J. Am. Chem. Soc., 2005, 127, 4128-4129; (h) H. Tang, R. J. Doerksen, T. V. Jones, M. L. Klein and G. N. Tew, Chem. Biol., 2006, 13, 427-435; (i) I. Ivanov, S. Vemparala, V. Pophristic, K. Kuroda, W. F. DeGrado, J. A. McCammon and M. L. Klein, J. Am. Chem. Soc., 2006, 128, 1778-1779; (j) K. Lienkamp, A. E. Madkour, A. Musante, C. F. Nelson, K. Nusslein and G. N. Tew, J. Am. Chem. Soc., 2008, 130, 9836-9843; (k) G. J. Gabriel, J. G. Pool, A. Som, J. M. Dabkowski, E. B. Coughlin, M. Muthukumar and G. N. Tew, Langmuir, 2008, 24, 12489-12495; (l) G. J. Gabriel, J. A. Maegerlein, C. E. Nelson, J. M. Dabkowski, T. Eren, K. Nusslein and G. N. Tew, Chem. - Eur. J., 2009, 15, 433-439; $(m)$ K. Lienkamp, A. E. Madkour, K. N. Kumar, K. Nusslein and G. N. Tew, Chem. - Eur. J., 2009, 15, 11715-11722; (n) K. Lienkamp and G. N. Tew, Chem. Eur. J., 2009, 15, 11784-11800; (o) B. P. Mowery, A. H. Lindner, B. Weisblum, S. S. Stahl and S. H. Gellman, J. Am. Chem. Soc., 2009, 131, 9735-9745; (p) K. Kuroda, 
G. A. Caputo and W. F. DeGrado, Chem. - Eur. J., 2009, 15, 1123-1133; $(q)$ G. N. Tew, R. W. Scott, M. L. Klein and W. F. Degrado, Acc. Chem. Res., 2010, 43, 30-39.

38 (a) J. Lin, S. Qiu, K. Lewis and A. M. Klibanov, Biotechnol. Bioeng., 2003, 83, 168-172; (b) A. M. Klibanov, J. Mater. Chem., 2007, 17, 2479-2482.

39 S. Krishnan, R. J. Ward, A. Hexemer, K. E. Sohn, K. L. Lee, E. R. Angert, D. A. Fischer, E. J. Kramer and C. K. Ober, Langmuir, 2006, 22, 11255-11266.
40 H. Nikaido and T. Nakae, Adv. Microb. Physiol., 1979, 20, 163-250.

41 H. J. Busscher and H. C. van der Mei, PLoS Pathog., 2012, 8, e1002440.

42 S. Ye, P. Majumdar, B. Chisholm, S. Stafslien and Z. Chen, Langmuir, 2010, 26, 16455-16462.

43 G. J. Gabriel, J. A. Maegerlein, C. F. Nelson, J. M. Dabkowski, T. Eren, K. Nusslein and G. N. Tew, Chem. - Eur. J., 2009, 15, 433-439. 